\title{
Volume Estimation of Small Particles Using Three-Dimensional Reconstruction from Multiple Views
}

\author{
Eduardo Castillo-Castaneda ${ }^{1}$ and Christelle Turchiuli ${ }^{2}$ \\ ${ }^{1}$ Instituto Politécnico Nacional, CICATA, \\ Cerro Blanco 141, Colinas del Cimatario, 76090, \\ Querétaro, Mexico \\ ecastilloca@ipn.mx \\ ${ }^{2}$ Agroparistech, UMR GenIAl 1145, 1 avenue des Olympiades, \\ 91744 Massy, France \\ christelle.turchiuli@agroparistech.fr
}

\begin{abstract}
This work presents a methodology and experimental results about the three dimensional reconstruction of small objects from an image sequence. The three dimensional model obtained from this methodology allows the volume estimation of particles less than $1 \mathrm{~mm}$. More precisely, the particles studied were generated by an agglomeration process from milk powder. To validate this methodology, a mechanical device with six degrees of freedom was built; it allows the montage of the image acquisition system as well as the turning system that rotates the particles. The algorithm for image processing and volume estimation was implemented using a high level language.
\end{abstract}

Keywords: Volume estimation, Three-dimensional reconstruction, Multiple views.

\section{Introduction}

A large quantity of food products are produced or used in the form of agglomerates, appealing for its ability to "solubility." This is a mechanical property that seeks to improve the product characteristics by changing parameters of the manufacturing process. The process of the agglomeration is to combine individual particles (a few hundred microns in size) to alter the shape of agglomerate and increase the porosity, then improving the solubility and the ability of powder rehydration, facilitating the penetration of the liquid inside the agglomerate.

The methods so far used to characterize the shape of the agglomerates are based on visualization through microscopes connected to computers to obtain digital images of the particles agglomerated [1]. A highly accurate technique is the use of confocal microscope [2] which is an optical imaging technique used to increase micrograph contrast and / or to reconstruct three-dimensional images by using a spatial pinhole to eliminate out-of-focus light or flare in specimens that are thicker than the focal plane.

Measuring open porosity by mercury porosimetry, which requires the application of high pressure, it is not suitable for the characteristics of agglomerates normally fragile 
that can break during measurement. The open porosity $\mathcal{E}_{a g g}$ can be also obtained from the volume envelope $V_{\text {env }}$, the mass $m$, and the density $\rho$ of agglomerate:

$$
\varepsilon_{\text {agg }}=1-\frac{m}{\rho \cdot V_{\text {env }}} .
$$

The volume calculation of irregularly shaped objects is usually solved by using the principle of Archimedes. However, the conventional methods fail when it comes to porous or fragile objects that cannot be subjected to external forces without altering their mechanical properties. In this case, methods of measuring volume without contact should be evaluated. A natural choice is the use of a computer-vision system that estimates the volume from a three-dimensional reconstruction of the object.

The work presented in [3] provides a rough measurement of the volume and the shape of agglomerates. The particle is placed in a turning table and the camera performs three shots at $0^{\circ}, 120^{\circ}$ and $240^{\circ}$. Using orthogonal projections, an average projected area of the particle is computed, then the volume of the sphere corresponding to that average area. The accuracy of this method is low because it assumes that the particles are spherical, which is not usually the case.

There are different approaches for three-dimensional reconstruction. In the most common case, it comes to getting the depth of a scene from two or more bidimensional images by matching techniques

The paper in [4] investigates a practical heuristics method for reconstruction of structured scenes from two uncalibrated images. This technique is used solely for the purpose of visualization a three-dimensional surface but not for purposes of calculating the volume since the information input (two images) is quite poor.

Some algorithms have been developed for modeling complex shaped objects using multiple views from image sequences [5], [6], [7]. Those works are mainly devoted to generate textured 3D models from real objects for applications such as virtual reality, computer animation and TV broadcasting. However, none of them have been particularly designed for volume measuring of small particles as the agglomerates concerned in this work.

This work presents an approach to calculate the open porosity; it consists on volume envelope estimation through three-dimensional reconstruction from multiples views of an agglomerate.

\section{Methodology}

The $3 \mathrm{D}$ reconstruction method presented here is based on the projection of multiple views of the object's silhouette to an initial volume cube that surely contains the object. The agglomerate turns on a rotary positioning device while a calibrated camera acquires an image sequence at fixed angular increments, as shown in Fig. 1. The particle turns around z-axis while $N$ images are acquired at angle $\theta_{i}, i=1, \ldots, N$. The angular increment $\alpha$ between each view is: $\alpha=\theta_{i+1}-\theta_{i}$.

The images are saved in JPG format. A backlight illumination provides an easy way to extract the silhouettes from images by conventional thresholding. 


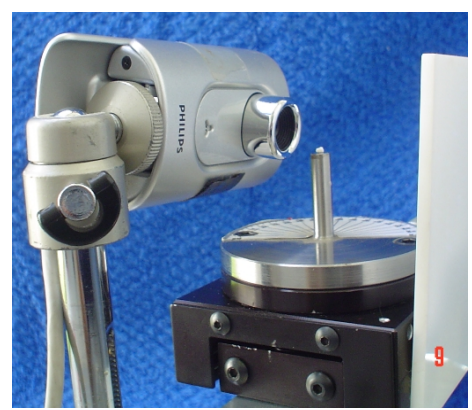

a)

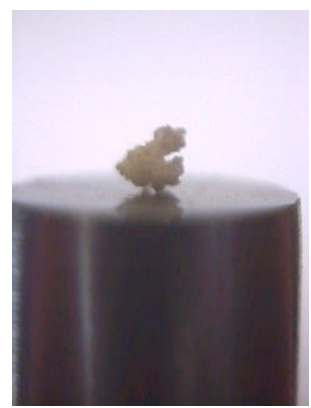

b)

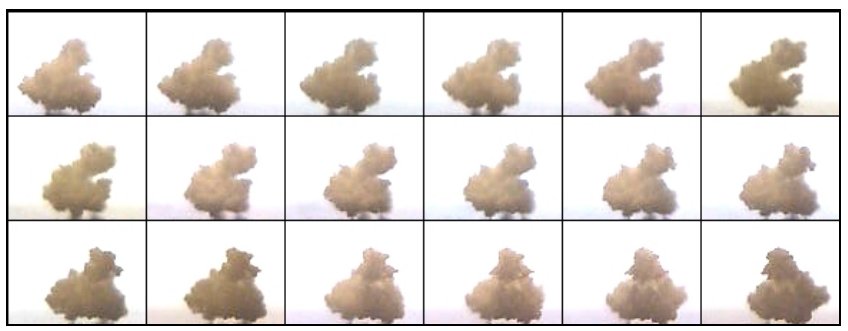

c)

Fig. 1. a) Rotary positioning device and usb camera, b) one agglomerate zoomed view, c) multiple views at fixed angular increments (10 degrees)

The silhouette of the $i$ image is described by a set of two-dimensional coordinates where $S_{i}=\left(y s_{i . j}, z s_{i, j}\right)$ is the number of black pixels that belong to the silhouette. The relationship that describes the three-dimensional coordinates of the points that belong to the projection $P_{i}=\left(x p_{i . j}, y p_{i . j}, z p_{i, j}\right)$ of the silhouette $i$ is (considering a rotation of $\theta_{i}$ ):

$$
\left[\begin{array}{c}
x p_{i, j} \\
y p_{i, j} \\
z p_{i, j}
\end{array}\right]=\left[\begin{array}{c}
x s_{i, j} \\
y s_{i, j} \\
z s_{i . j}
\end{array}\right]\left[\begin{array}{ccc}
\cos \theta_{i} & -\sin \theta_{i} & 0 \\
\sin \theta_{i} & \cos \theta_{i} & 0 \\
0 & 0 & 1
\end{array}\right] .
$$

The coordinates of the points belonging to the volume $V_{i}=\left(x v_{i, j, k}, y v_{i, j, k}, z v_{i, j, k}\right)$ are generated by $M$ translations $T_{k}, k=1, \ldots, M$, that are normal to the plane of the projection $P_{i}$, it means:

$$
\left[\begin{array}{c}
x v_{i, j, k} \\
y v_{i, j, k} \\
z v_{i, j, k}
\end{array}\right]=\left[\begin{array}{c}
x p_{i, j} \\
y s_{i, j} \\
z s_{i, j}
\end{array}\right]+\left[\begin{array}{c}
k \lambda \sin \left(\theta_{i}+\pi / 4\right) \\
k \lambda \cos \left(\theta_{i}+\pi / 4\right) \\
0
\end{array}\right] .
$$


Where $\lambda$ is a constant step equal to 10 to ensure enough density in the generated volume. Thus, the total volume is given by the intersection of $N$ partial volumes $V i$ :

$$
V_{T}=\bigcap_{i=1, \ldots, N} V_{i} .
$$

Since the silhouette projection is a pixel-based algorithm, the volume is given simply by counting the number of voxels belonging to the intersection. For a three dimensional visualization of the reconstructed agglomerates, the volume is presented as a cloud of points in the Cartesian space. A triangular mesh using the Delaunay triangulation that is a set of lines connecting each point to its natural neighbors approximates the surface of the volume model.

As an example, the Fig. 2a shows a silhouette of the Eiffel Tower. The volume generated for the projection at 0 degrees is shown in Fig. 2b. Two volumes generated at 0 and 90 degrees are shown in Fig. 2c. Finally, the three-dimensional reconstruction is shown in Fig. 2 d. In this example the number of black pixels in each silhouette is 6975 ; the total volume is 284628 voxels.

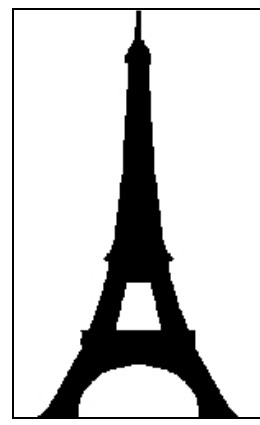

a)

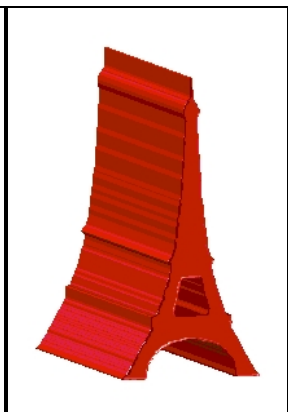

b)

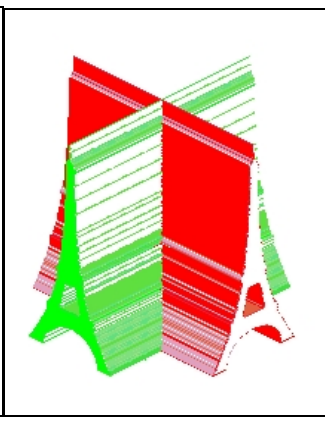

c)

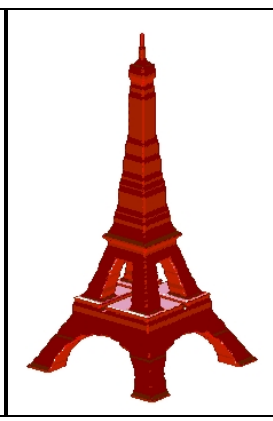

d)

Fig. 2. a) Silhouette b) Projection and its translation, c) Two projections, d) Three-dimensional reconstruction

\section{Numerical Validation}

The technique of volumes intersection was implemented in c++ language. That implementation was validated using the image of a circle of 100 pixels in diameter supposed to be the image of a sphere of 523600 voxels volume. The advantage of such object is that image remains unchanged for any rotation angle of the object. Thus, it was possible to validate the accuracy of the method depending on the number of images acquired. Some of the three-dimensional images obtained are presented in Fig. 3.

Actually, there is very little difference in the quality of reconstruction for more than 20 images. Table 1 presents the estimated volumes depending on the number of images acquired, including the error of the estimation considering the actual volume of a sphere diameter of 100 pixels (actual volume equal to 523600 voxels).

The table shows that the percentage of error for more than 20 images is less than $1 \%$. The error for 180 images (views with angular increments of 1 degree) is not very much different for 60 images (views with angular increments of 3 degrees). 


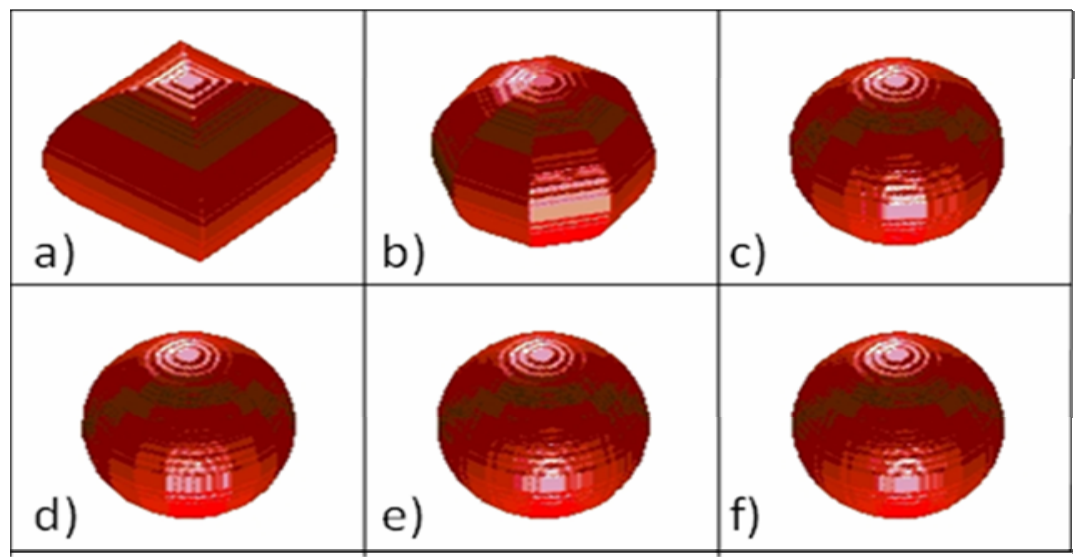

Fig. 3. Three-dimensional reconstruction with different number of images, a) 2 , b) 4 , c) 8 , d) 10 , e) 20, f) 60

Table 1. Estimated volume and error depending on the number of images

\begin{tabular}{|c|c|c|c|}
\hline $\begin{array}{c}\text { No. of } \\
\text { images }\end{array}$ & $\begin{array}{c}\text { Computed } \\
\text { volume } \\
\text { (voxels) }\end{array}$ & $\begin{array}{c}\text { Error } \\
\text { (voxels) }\end{array}$ & $\begin{array}{c}\text { Error } \\
(\%)\end{array}$ \\
\hline 2 & 667,368 & 143,768 & 27.46 \\
\hline 4 & 554,892 & 31,292 & 5.98 \\
\hline 6 & 538,976 & 15,376 & 2.94 \\
\hline 8 & 533,486 & 9,886 & 1.89 \\
\hline 10 & 530,784 & 7,184 & 1.37 \\
\hline 20 & 527,404 & 3,804 & 0.73 \\
\hline 30 & 526,932 & 3,332 & 0.64 \\
\hline 60 & 526,056 & 2,456 & 0.47 \\
\hline 90 & 525,852 & 2,252 & 0.43 \\
\hline 180 & 525,740 & 2,140 & 0.41 \\
\hline
\end{tabular}

\section{Experimental Validation and Results}

To validate the performances of the angular positioning device and extraction of silhouette from actual images, one used a plastic soldier $2.5 \mathrm{~cm}$ in height. In this case, 18 images were used, that means angular increments of 10 degrees. The size of the images was 102x63 pixels. A backlight illumination provides good contrast between object and background that simplifies the silhouettes extraction from images. Fig. 4 presents the 18 views and the respective silhouettes extracted.

Fig. 5 presents the three-dimensional reconstruction obtained; Fig. 5a shows that there is a minimal loss of object features. A volume of 128879 voxels was obtained, whereas $1 \mathrm{~mm}$ in the actual image is equivalent to 6.7 pixels, the volume estimated is 


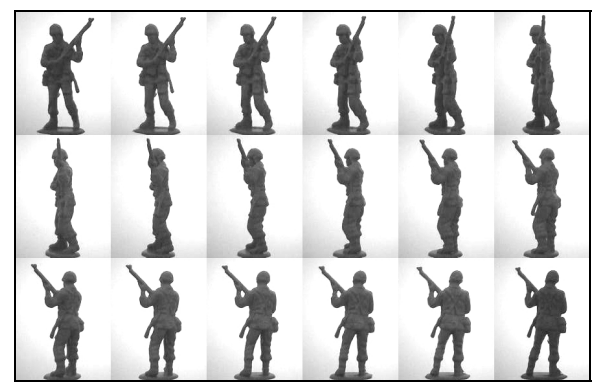

a)

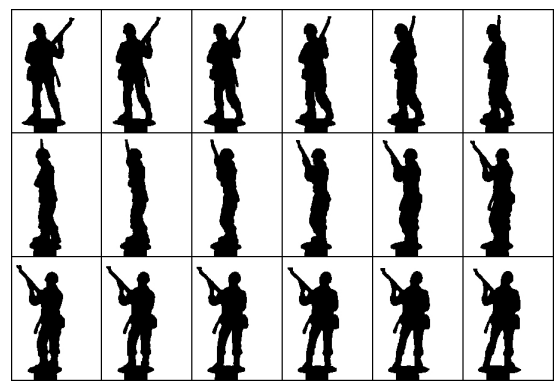

b)

Fig. 4. a) Views of the object, b) Extracted silhouettes

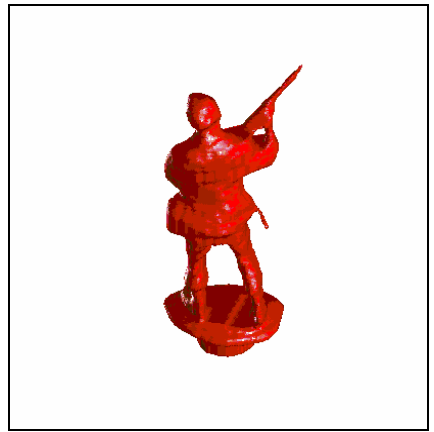

a)

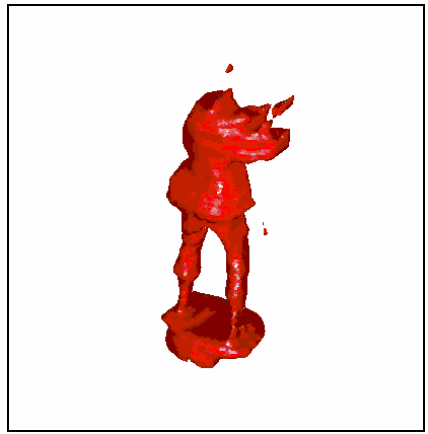

b)

Fig. 5. a) Three-dimensional reconstruction, c) faulty reconstruction by an error in the positioning system

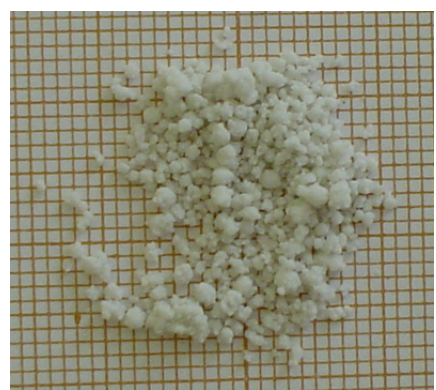

a)

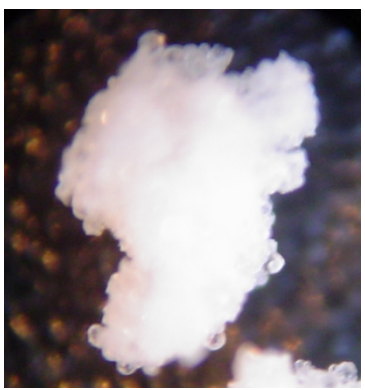

b)

Fig. 6. a) Agglomerate sample on graph paper, b) Zoomed view of one agglomerate

$428 \mathrm{~mm} 3$. Using the principle of Archimedes, the actual volume of the object was $410 \mathrm{~mm} 3$. In Fig. $5 \mathrm{~b}$ there is a faulty reconstruction obtained by an error in the orientation angle of the positioning system, causing the loss of some features of the object. 
The methodology was tested in milk agglomerated particles, the size of the samples and its structure can be seen in Fig. 6.

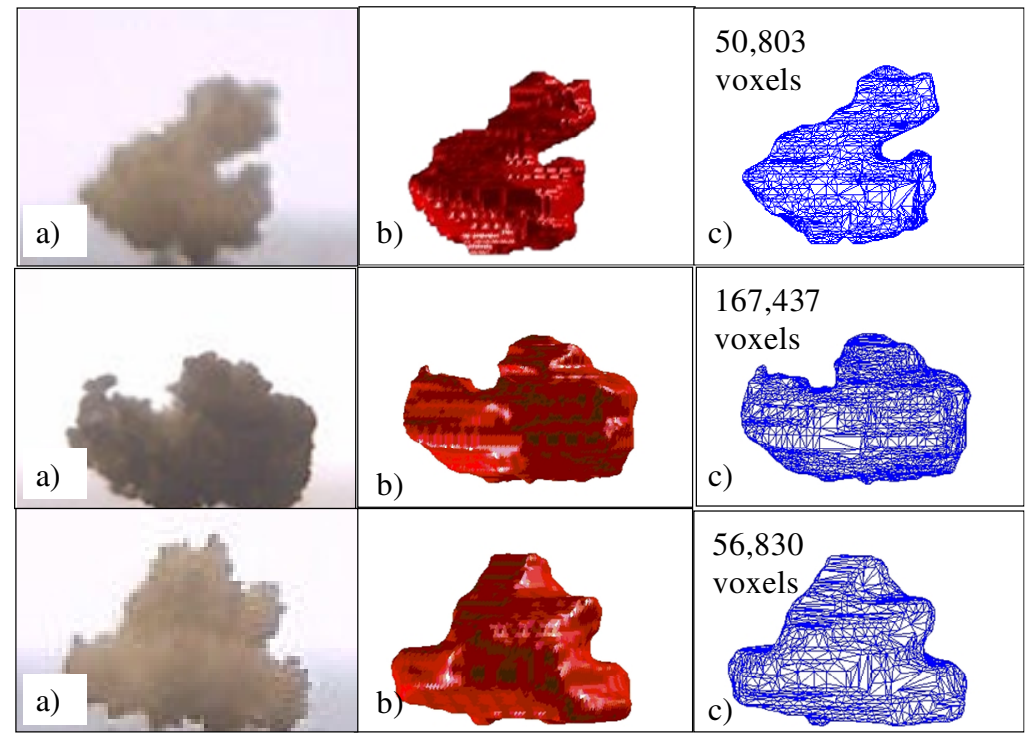

Fig. 7. Three different agglomerates: a) Actual object, b) 3D reconstruction, c) triangular mesh and volume

The Fig. 7 presents some agglomerates, their three-dimensional reconstructed models, and the estimated volume.

\section{Conclusions}

This paper presents an experimental validation of a technique for three-dimensional reconstruction of small particles from processing a sequence of images. The reconstruction allows the calculation of the volume of the particles.

The object digitalization generates loss of accuracy of the method, however, as demonstrated in the case of the reconstruction of the sphere, the error generated is less than $1 \%$ for a number of images around 20; the foregoing allowed to consider only 18 images, that is views with an angular increment of 10 degrees.

The calculation of each projected image on the candidate volume requires a very precise knowledge of the rotation axis position. To find precisely that position, two views of the object were used ( 0 and 180 degrees). The composition of both views should form a symmetrical image; the position of the axis of symmetry corresponds to the position of the rotation axis of the positioning system.

The size of the images is considered important in the final resolution of this method. The total amount of memory available on the PC where the experiments were conducted, limited the image size to $250 \times 250$ pixels. 


\section{References}

1. Turchiuli, C., Eloualia, Z., El-Mansouri, N., Dumoulin, E.: Fluidised bed agglomeration: Agglomerates shape and end-user properties, Internal report ENSIA (2005)

2. Pawley, J.B.: Handbook of Biological Confocal Microscopy, 3rd edn. Springer, Berlin (2006)

3. Hogekamp, S., Pohl, M.: Porosity measurement of fragile agglomerates, Powder Technology, pp. 385-392 (2003)

4. Wang, G., Tsui, H.T., Hu, Z.: Reconstruction of structured scenes from two uncalibrated images. Pattern Recognition Letters 26(2), 207-220 (2005)

5. Fang, Y.H., Chou, H.L., Chen, Z.: 3D shape recovery of complex objects from multiple silhouette images. Pattern Recognition Letters 24(9-10), 1279-1293 (2003)

6. Fitzgibbon, A.W., Cross, G., Zisserman, A.: Automatic 3D Model Construction for Turn Table Sequences. In: Proceedings of the European Workshop on 3D Structure from Multiple Images of Large-Scale Environments, pp. 155-170 (1998)

7. Niem, W., Buschmann, R.: Automatic Modelling of 3D Natural Objects from Multiple Views. In: European Workshop on Combined Real and Synthetic Image Processing for Broadcast and Video Production, Hambourg, Germany (1994) 